\title{
A NOVEL ALGORITHM OF KINEMATICS PARAMETERS MEASUREMENT FOR UPPER LIMB BASED ON MOTION CAPTURE
}

\author{
Shuai Guo ${ }^{1}$, Xuesheng Xiong ${ }^{1}$, Leigang Zhang ${ }^{1}$, Qing Sun ${ }^{1}$, \\ ${ }^{1}$ Shanghai Key Laboratory of Intelligent Manufacturing and Robotics, Shanghai University, \\ No.99 Shangda Road, Baoshan District, Shanghai 200444, China \\ E-mails: guoshuai@shu.edu.cn,18717923650@163.com, Igzhang@shu.edu.cn, qingsun93@163.com
}

\begin{abstract}
Joint position and joint angle are the basic kinematic parameters for the quantitative evaluation of upper limb motion ability, which are widely applied to calculate extremity reachable workspace and range of motion (ROM). The traditional measurement methods cannot meet the clinical requirements in accuracy, stability and real-time. This paper proposes a novel algorithm for measuring the kinematic parameters of upper limb by combining the kinematic chain method with the vector projection method. Firstly, the human upper limb motion model is simplified to a 7 degrees of freedom (DOF) motion model according to the anatomical principle, and the rigid body group is used to collect the upper limb kinematic parameters to realize the real-time tracking of trajectory and angle of the shoulder, elbow and wrist joints. Finally, a healthy volunteer experiment is designed, the results show that the algorithm can provide real-time and quantitative upper limb kinematic parameters, which can meet the data requirements of quantitative evaluation of upper limbs.
\end{abstract}

Keywords: Upper Limb Joint, Kinematic Parameters, Kinematic Chain, Vector Projection, Motion Capture.

\section{Introduction}

Researches have shown that upper limb movements of stroke patients are characterized by decreased movement coordination and increased movement variability and segment [1]. Therefore, changes in muscle strength and changes in holistic motion mode are widely used as criteria for evaluation of upper limb motor function [2]. Traditional scale evaluation relies too much on the subjective of physicians, which leads to the difficulty of accurate quantification of results [3]. Therefore, the quantitative evaluation method based on the characteristics of upper limb kinematics parameters is gradually adopted by researchers. These studies achieve the purpose of quantitative evaluation of upper limb motor function through setting up evaluation indexes which are based on the kinematics parameters collected in some training tasks [4].

Joint position and joint angle are the main basic of upper limb kinematic parameters [5][6]. Clinically, the universal protractor and photographic methods are commonly used to measure these kinematic parameters, but these methods cannot meet the clinical needs in terms of accuracy, stability and realtime requirement [7]. With the development of sensor technology, researchers use various sensors combined with corresponding algorithms to calculate these kinematic parameters. For instance,
Chen at al. [8] propose a multi-rigid real-time tracking system based on the upper limb kinematic chain algorithm to achieve shoulder and elbow joint angle measurement, in which Kalman filter is used to improve the system robustness, while the algorithm does not involve the measurement of the wrist joint kinematic parameters. Since wrist joint injury has a great influence on the fine movement of the hand and even the activity of the upper limb [9], it is indispensable to distinguish the degree of wrist joint injury and adopt effective rehabilitation methods in time [10]. Nguyen at el. [11] present a sensing system based on optical linear encoder (OLE), in which the motion of an optical encoder on a code strip is converted to goniometric data of the limb joints. However, this algorithm does not involve the measurement of joint position, and only applies to specific sensors. Song at el. [12] propose a method of measuring upper limb motion parameters by converting joint relative pose matrix to Euler angle based on electromagnetic tracking system, but the calculation results are related to the selected rotation order of the Euler angle, so the calculation results may be different from the actual joint angle. In addition, scholars use Microsoft Kinect to measure the kinematics parameters of upper limbs [13-15]. After measuring joint position data by this system, vector projection method is used to calculate joint angle, while this measurement algorithm is complex and its accuracy needs to be improved. 
A novel algorithm combining kinematic chain method and vector projection method is proposed in this paper according to the above analysis to measure kinematic parameters of upper limbs in order to solve the shortcomings of the existing methods. The 7 groups rigid bodies are used to acquire the kinematic parameters of the upper limb links to establish the pose matrix of each joint coordinate system, and calculate the position and angle of shoulder, elbow and wrist joints in real time by combining the kinematic chain method and vector projection method.

\section{Upper Limb Kinematics Model}

The upper limb is the most flexible system in the motion system of various parts of the human body. From the perspective of mechanical kinematics, the upper limb is a multi-degree-of-freedom mechanical motion system with many complex structural joints [16], which should be simplified on the premise of rigid body hypothesis theory when analyzing the upper limb's motion ability. 7DOF model is the most universal model [17], since this model is the closest to the motion function of human upper limbs, which can well describe human motion [8]. In this model, shoulder joint has three degrees of freedom: abduction and adduction, medial rotation and lateral rotation, flexion and extension, elbow joint has one freedom: flexion and extension, wrist joint has three degrees of freedom: dorsal extension and palmar flexion, supination and pronation, abduction and adduction. The simplified 7DOF kinematics model of upper limb is shown in Figure 1.

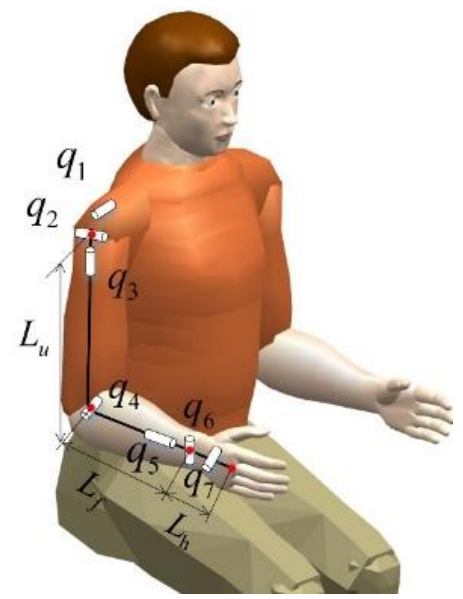

Figure 1: 7 DOF simplified model of upper limb kinematics.

\section{Method \\ 3.1. Static calibration algorithm}

Static calibration mainly completes the calculation of the transformation relationship between the joint coordinate system of upper limb and the corresponding rigid body coordinate system. The upper limb trunk, upper arm, forearm, and hand are regarded as rigid bodies according to the above 7 DOF simplified model, 7 groups of rigid bodies of dual-arm are connected by joints. The coordinate system of shoulder joint is fixed on trunk, the coordinate system of elbow joint is fixed on upper arm, the coordinate system of wrist joint is fixed on forearm, and the coordinate system of hand is fixed on the root of middle finger. The method of reference [12] is used to establish the coordinate system, the position and direction of each joint coordinate system are shown in Figure 2. The left and right joint coordinate systems are indicated respectively by dashed lines and solid lines, and the 7 sets of rigid bodies $\left(B_{1} \div B_{7}\right)$ are fixed on the outside of each link of the dual-arm so as to be recognized by the motion capture system to collect the motion parameters of each rigid body of the dual-arm.

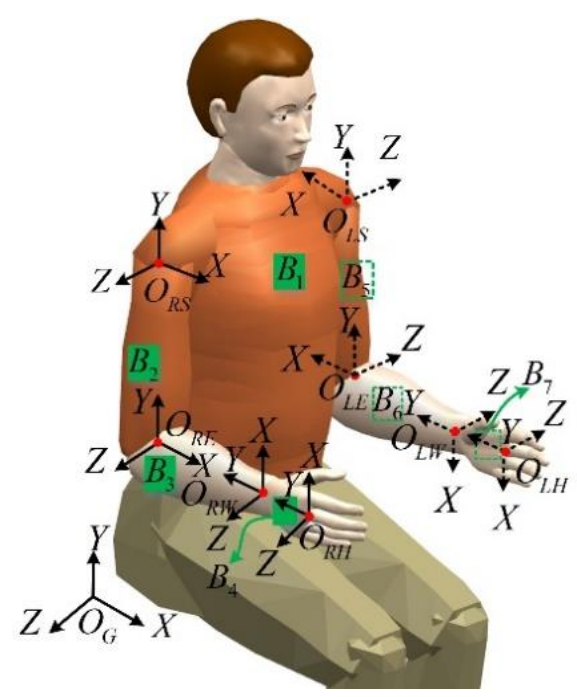

Figure 2: Upper limbs joint coordinate system and rigid body wearing method.

During the movement of the upper limbs, the 7 groups of rigid bodies and the corresponding joint coordinate system are always on the same rigid body. Therefore, the pose data of joint coordinate system can be obtained according to the data of the corresponding rigid body as long as the transformation relationship between the joint coordinate system and the corresponding rigid body coordinate system is obtained at the initial moment. In order to facilitate the acquisition of the anatomical position of the upper limb, this paper stipulates that the tester takes the sitting position at the initial moment, the upper arm is vertically downward, the forearm is at an angle of $90^{\circ}$ to the upper arm, the hand is straight, the hands of both sides are facing right, and the hand of the hand is inward, as shown in Figure 2. 
The position of acromion, medial epicondyle and lateral epicondyle, ulnar side, flexor side and middle finger root are measured by auxiliary rigid body $B_{0}$ for static calibration. And then calculate the position $O_{S}, O_{E}$ and $O_{W}$ of each joint coordinate system centre and direction of the shoulder joint, elbow joint and wrist joint coordinate system according to the calculation method in [12]. In addition, the origin of the hand coordinate system is fixed on the finger root of the middle finger, the initial position $O_{H}$ of the coordinate system center is measured by $B_{0}$ directly and the initial direction of this coordinate system is the same as the wrist joint coordinate system.

A set of data of 7 rigid bodies is obtained to calculate the transformation matrix between the rigid body coordinate system and the corresponding joint coordinate system after establishing the joint coordinate system at the initial time. The coordinate system of right and left shoulder joint both correspond to the coordinate system of $B_{1}$.

This paper takes right limb as an example to introduce the calculation method of the transformation relationship between rigid body coordinate system and corresponding joint coordinate system.

Firstly, the pose matrix of $B_{i}$ in the global coordinate system at the initial time is calculated :

$$
\begin{aligned}
{ }_{B_{i}}^{G} R_{t 0} & =R_{x_{i}}(x r o t) R_{y_{i}}(y r o t) R_{z_{i}}(z r o t) \\
{ }_{B_{i}}^{G} T_{t 0} & =\left(\begin{array}{cc}
{ }_{B_{i}}^{G} R_{t 0} & P_{B_{i}} \\
0 & 1
\end{array}\right)
\end{aligned}
$$

where $i=1,2,3,4$, xrot, yrot, zrot are the $X Y Z$ dynamic Euler angle of rigid body in the global coordinate system, $P_{B_{i}}$ is the position information of $B_{i}$ in the global coordinate system.

Secondly, the relative transformation matrix between the coordinate system of $B_{1}$ and shoulder joint coordinate system is calculated:

$$
{ }_{R S}^{B_{1}} T={ }_{R S}^{G} T_{0} \cdot\left({ }_{B_{1}}^{G} T_{t 0}\right)^{-1}
$$

where ${ }_{R S}^{G} T_{0}$ represents the pose matrix of the shoulder coordinate system in the global coordinate system, which is constructed from the position and direction vectors of the shoulder joint coordinate system. ${ }_{R S}^{B_{1}} T$ is the relative transformation matrix between the coordinate system of $B_{1}$ and shoulder coordinate system, which remains unchanged during upper limb movement.
According to above principle, the relative transformation matrices between other groups of joints coordinate system and corresponding rigid bodies coordinate system are obtained respectively, which include ${ }_{R E}^{B_{2}} T,{ }_{R W}^{B_{3}} T$ and ${ }_{R H}^{B_{4}} T$ :

$$
\begin{aligned}
& { }_{R E}^{B_{2}} T={ }_{R E}^{G} T_{0} \cdot\left({ }_{B_{2}}^{G} T_{t 0}\right)^{-1} \\
& { }_{R W}^{B_{3}} T={ }_{R W}^{G} T_{0} \cdot\left({ }_{B_{3}}^{G} T_{t 0}\right)^{-1} \\
& { }_{R H}^{B_{4}} T={ }_{R H}^{G} T_{0} \cdot\left({ }_{B_{4}}^{G} T_{t 0}\right)^{-1}
\end{aligned}
$$

\subsection{Kinematics parameter measurement algorithm}

After completing static calibration, the motion capture system acquires the pose parameters of 7 rigid bodies in real time during the upper limb movement.

\section{(1) Joint position calculation method}

Firstly, calculate the pose matrix of each rigid body in the global coordinate system.

$$
\begin{aligned}
{ }_{B_{i}}^{G} R & =R_{x i}(x r o t) R_{y i}(y r o t) R_{z i}(z \text { rot }) \\
{ }_{B_{i}}^{G} T & =\left(\begin{array}{cc}
{ }_{B_{i}}^{G} R & P_{B_{i}} \\
0 & 1
\end{array}\right)
\end{aligned}
$$

where $P_{B_{i}}$ is the position matrix of rigid body $B_{i}$ in global coordinate system. Then calculate the pose matrix of each joint coordinate system in the global coordinate system sequentially according to the pose transformation matrix between each rigid body coordinate system and the corresponding joint coordinate system.

$$
{ }_{R S}^{G} T={ }_{B_{1}}^{G} T \cdot{ }_{R S}^{B_{1}} T
$$

where ${ }_{R S}^{G} T$ is the pose matrix of the shoulder joint in the global coordinate system. The pose matrix ${ }_{R E}^{G} T$, ${ }_{R W}^{G} T$ and ${ }_{R H}^{G} T$ of the elbow joint, wrist joint and hand coordinate system in the global coordinate system are respectively calculated according to the equation (9):

$$
\begin{aligned}
& { }_{R E}^{G} T={ }_{B_{2}}^{G} T \cdot{ }_{R E}^{B_{2}} T \\
& { }_{R W}^{G} T={ }_{{ }_{3}}^{G} T \cdot{ }_{R W}^{B_{3}} T \\
& { }_{R H}^{G} T={ }_{B_{4}}^{G} T \cdot{ }_{R H}^{B_{4}} T
\end{aligned}
$$

Each pose matrix of joint coordinate system above contains the real-time position and posture information of the joint in the global coordinate system. 


\section{(2) Joint angle calculation method}

\section{A. Elbow joint}

The upper limb simplified kinematic chain is constructed according to the position information of the shoulder joint coordinate system, the elbow joint joint coordinate system and the wrist joint joint coordinate system obtained above, and as shown in Figure 3, the rotation angle of the elbow joint is obtained by the cosine theorem.

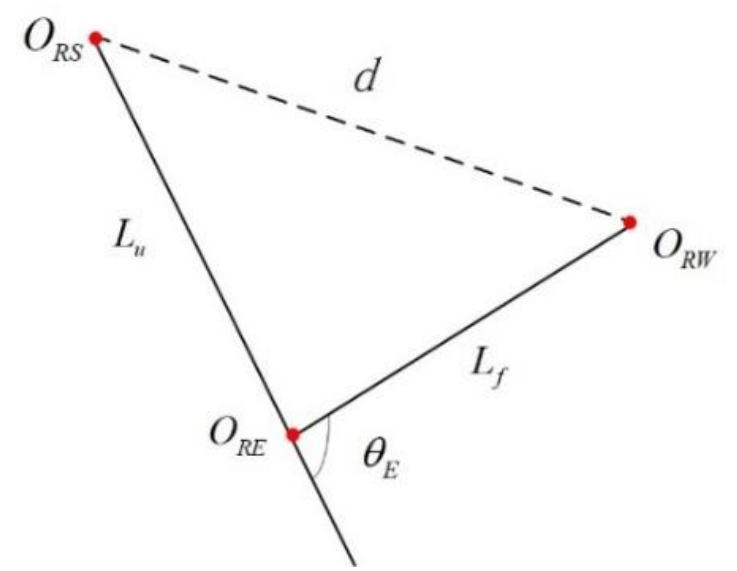

Figure 3: The upper limbs simplified kinematic chain

$$
\theta_{E}=\pi-\arccos \left(L_{u}^{2}+L_{f}^{2}-\mathrm{d}^{2}\right) /\left(2 \cdot L_{u} \cdot L_{f}\right)
$$

where $L_{u}, L_{f}$, represent the length of the upper arm and forearm respectively, and $\mathbf{d}$ is the distance between the shoulder joint centre and the wrist joint centre.

\section{B. Shoulder joint}

According to the pose matrix of the shoulder, elbow and wrist joint coordinate system obtained above, the pose matrix of the elbow joint and the wrist joint coordinate system in the proximal coordinate system of the shoulder joint can be obtained:

$$
\begin{aligned}
& { }_{R E}^{R S} T={ }_{R E}^{G} T \cdot\left({ }_{R S}^{G} T\right)^{-1} \\
& { }_{R W}^{R S} T={ }_{R W}^{G} T \cdot\left({ }_{R S}^{G} T\right)^{-1}
\end{aligned}
$$

where ${ }_{R E}^{R S} T$ is the relative pose matrix of the elbow joint coordinate system in the shoulder joint coordinate system, which includes the position $E_{s} \cdot x, E_{s} \cdot y$ and $E_{s} \cdot z$ of the elbow joint centre in the shoulder joint coordinate system, ${ }_{R W}^{R S} T$ is the relative pose matrix of the wrist joint coordinate system in the shoulder joint coordinate system, which include the position $W_{s} \cdot x, W_{s} \cdot y$ and $W_{s} \cdot z$ of the wrist joint centre in the shoulder joint coordinate system.

The rotation of the upper arm around the y-axis of the shoulder joint coordinate system does not change the position of the elbow joint centre under the shoulder joint coordinate system during the movement of the upper limbs. It is assumed that the rotation order of the upper arm around the shoulder joint coordinate system is $Z-X-Y$, the angle of the shoulder joint around the $\mathrm{x}$-axis of its coordinate system the angle around the z-axis of its coordinate system can be obtained from the equation (16).

$$
\begin{gathered}
\boldsymbol{T}_{s z} \cdot \boldsymbol{T}_{s x} \cdot \boldsymbol{T}_{s e} \cdot\left(\begin{array}{llll}
0 & 0 & 0 & 1
\end{array}\right)^{T} \\
=\left(\begin{array}{llll}
E_{s} \cdot x & E_{s} \cdot y & E_{s} \cdot z & 1
\end{array}\right)^{T} \\
\left(\begin{array}{cccc}
\cos \theta_{s z} & -\sin \theta_{s z} & 0 & 0 \\
\sin \theta_{s z} & \cos \theta_{s z} & 0 & 0 \\
0 & 0 & 1 & 0 \\
0 & 0 & 0 & 1
\end{array}\right)\left(\begin{array}{cccc}
1 & 0 & 0 & 0 \\
0 & \cos \theta_{s x} & -\sin \theta_{s x} & 0 \\
0 & \sin \theta_{s x} & \cos \theta_{s x} & 0 \\
0 & 0 & 0 & 1
\end{array}\right) \\
\left(\begin{array}{cccc}
1 & 0 & 0 & 0 \\
0 & 1 & 0 & -L_{u} \\
0 & 0 & 1 & 0 \\
0 & 0 & 0 & 1
\end{array}\right)\left(\begin{array}{l}
0 \\
0 \\
0 \\
1
\end{array}\right)=\left(\begin{array}{llll}
E_{s} \cdot x & E_{s} \cdot y & E_{s} \cdot z & 1
\end{array}\right)^{T}
\end{gathered}
$$

hence:

$$
\begin{aligned}
& \left(\begin{array}{llll}
L_{u} \cdot \sin \theta_{s z} \cos \theta_{s x} & -L_{u} \cdot \cos \theta_{s z} \cos \theta_{s x} & -L_{u} \cdot \sin \theta_{s x} & 1
\end{array}\right)^{T} \\
& =\left(\begin{array}{llll}
E_{s} \cdot x & E_{s} \cdot y & E_{s} \cdot z & 1
\end{array}\right)^{T}
\end{aligned}
$$$$
\sin \theta_{s x}=-\frac{E_{s} \cdot z}{L_{u}}
$$$$
\cos \theta_{s x}= \pm \frac{\sqrt{\left(E_{s} \cdot x\right)^{2}+\left(E_{s} \cdot y\right)^{2}}}{L_{u}}
$$

For $\theta_{s x}$, one set of solutions is

$$
\theta_{s x}=a \tan 2\left(-E_{s} \cdot z, \sqrt{\left(E_{s} \cdot x\right)^{2}+\left(E_{s} \cdot y\right)^{2}}\right)
$$

another set of solutions is:

$$
\theta_{s x}^{\prime}=\left\{\begin{array}{lr}
\pi-\theta_{s x} & \left(\text { if } \sin \theta_{s x}>0\right) \\
-\pi-\theta_{s x} & \text { (else) }
\end{array}\right.
$$

Similarly, for $\theta_{s z}$, one set of solutions is:

$$
\begin{aligned}
& \theta_{s z}=a \tan 2\left(E_{s} \cdot x,-E_{s} \cdot y\right) \\
& \theta_{s z}^{\prime}=a \tan 2\left(-E_{s} \cdot x, E_{s} \cdot y\right)
\end{aligned}
$$


When calculating the final solution of $\theta_{s x}$ and $\theta_{s z}$ it needs to be compared with the result of the previous moment and select the set of solutions with a small amount of change as the final solution.

Similarly, the angle of the shoulder joint around the y-axis of its coordinate system can be obtained by equation (25).

$$
\begin{aligned}
& T_{s z} \cdot T_{s x} \cdot T_{s y} \cdot T_{s e} \cdot T_{e} \cdot T_{e w} \cdot\left(\begin{array}{llll}
0 & 0 & 0 & 1
\end{array}\right)^{T} \\
& =\left(\begin{array}{lllll}
W_{s} \cdot x & W_{s} \cdot y & W_{s} \cdot z & 1
\end{array}\right)^{T}
\end{aligned}
$$

where $T_{e}$ is the elbow joint coordinate system rotation matrix, $T_{e w}$ is the translation matrix of the elbow joint coordinate system to the wrist joint coordinate system.

\section{Wrist joint}

The normal 3 groups ROM of wrist joint angles are all within $\left[-90^{\circ}, 90^{\circ}\right]$, and the numerical value is the deviation of the hand coordinate system from the wrist joint coordinate system. Therefore, the rotation angle $\theta_{w x}, \theta_{w y}$ and $\theta_{w z}$ of the wrist joint can be calculated directly by the coordinate vector projection method.

$$
\theta_{w x}=\overrightarrow{h w} \cdot \overrightarrow{w y} /(|\overrightarrow{h w}||\overrightarrow{w y}|)
$$

where $\overrightarrow{h w}$ is the projection of the vector formed by hand coordinate system centre to the wrist joint coordinate system centre on the $Y O Z$ plane in the wrist coordinate system, $\overrightarrow{w y}$ is the directional vector of the $y$-axis of the wrist joint coordinate system.

$$
\theta_{w y}=\overrightarrow{h x} \cdot \overrightarrow{w x} /(|\overrightarrow{h x}||\overrightarrow{w x}|)
$$

where $\overrightarrow{h x}$ is the projection of the $\mathrm{x}$-axis directional vector of the hand coordinate system on the $Y O Z$ plane of the wrist coordinate system, $\overrightarrow{w x}$ is the directional vector of the $\mathrm{x}$-axis of the wrist joint coordinate system.

$$
\theta_{w z}=\overrightarrow{h w} \cdot \overrightarrow{w y} /(|\overrightarrow{h w}||\overrightarrow{w y}|)
$$

where $\overrightarrow{h w}$ ' is the projection of vector formed by hand coordinate system centre to the wrist joint coordinate system centre on the $X O Y$ plane in the wrist coordinate system, $\overrightarrow{w y}$ is the directional vector of the $y$-axis of the wrist joint coordinate system.
The algorithm above provides the calculation method of shoulder, elbow and wrist joint centre position and 7 DOF joint angle. The positive and negative values represent the direction of rotation when calculating the wrist joint angle, the value is positive when it is rotated clockwise along the positive direction of the coordinate axis specified in the paper.

\section{Experiments and Results}

This paper takes the right arm as an example to performs single degree of freedom experiment and activities of daily living (ADL) motion experiment respectively to judge whether the algorithm calculation result is in line with the actual motion situation.

\subsection{Experimental equipment}

The optical motion capture system is the most accurate motion capture system which is usually used as a measurement standard to verify the accuracy of other motion capture systems [18][19]. The Optitrack optical motion capture system is used in this study to verify the correctness of the algorithm. Each rigid body is constructed by multiple markers that can be recognized by cameras, the rigid body $B_{1} \sim B_{7}$ is fixed to the experimenter by a bandage as shown in Figure 4.

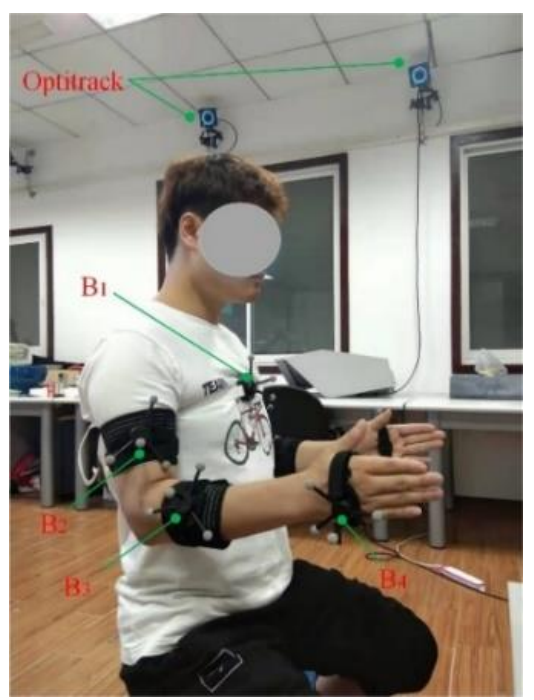

Figure 4: Optitrack motion capture system and rigid bodies, $B_{4} \sim B_{7}$ are worn on the left arm which are not shown in this figure

\subsection{Subjects and experimental procedure}

A healthy male volunteer without any dyskinesia with the age of 23 years is included in this experiment. The volunteer is asked to perform single degree of freedom experiment exercise experiment and ADL motion experiment. 
The single degree of freedom rotational motion of the shoulder, elbow, and wrist joints is performed in the experiment, and the subject is required to return to the initial position after the reciprocating single joint motion from the static calibration initial position. ADL-based exercise ability assessment is widely used in sports, injury, rehabilitation and joint flexibility evaluation [20].

This paper selects the water pouring action to analyse the kinematic parameters of the upper limbs, which requires the subject to proceed from the initial position, and then complete a series of actions of taking a cup, pouring water, and returning to the initial position.

\subsection{Result}

A. The angle curve of each joint in the single joint motion experiment is shown in Figures 5, 6 and 7.

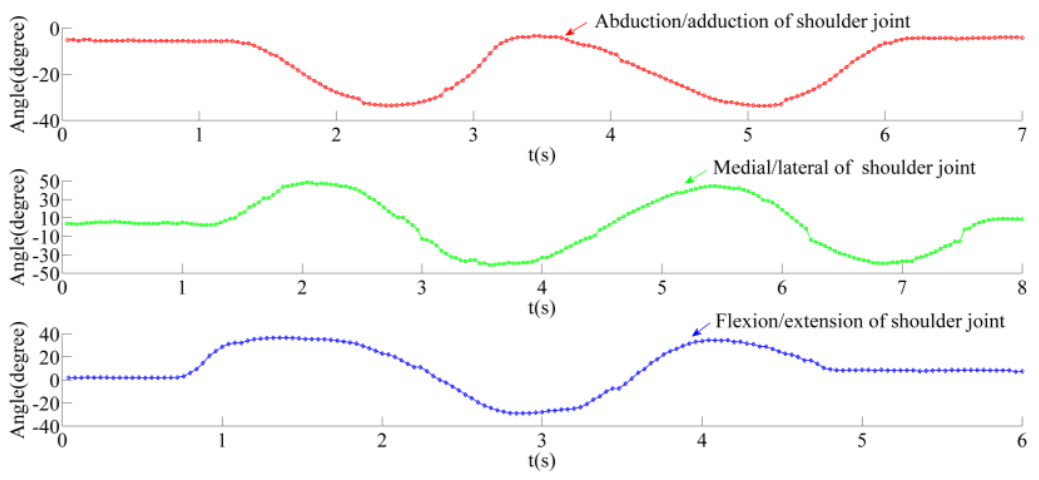

Figure 5: Shoulder joint angle of the single degree of freedom experiment, these curves show obvious periodicity.

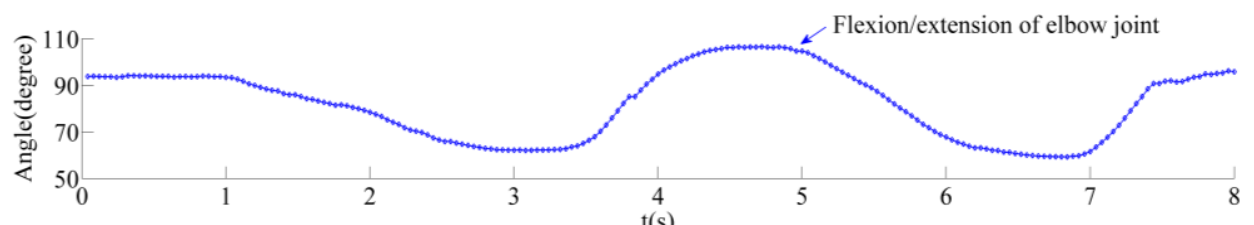

Figure 6: Elbow joint angle of the single degree of freedom experiment, the elbow initial angle approached $90^{\circ}$ and finally returns to the initial position.

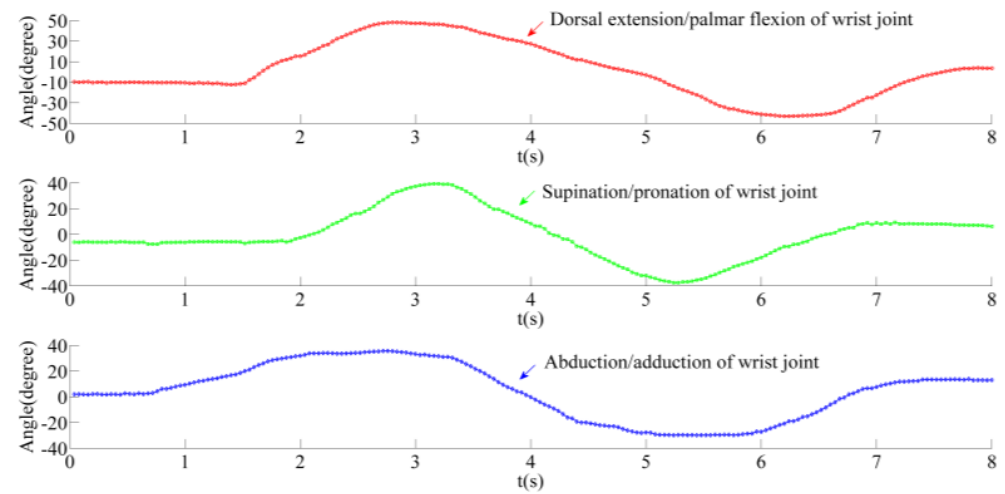

Figure 7: Wrist joint angle of the single degree of freedom experiment, these curves also show obvious periodicity.

The results of single joint motion experiments show that the joint angle changes of the 7 groups show obvious periodicity, which is in line with the actual motion. The joint angle change curve is close to the sine function. The occurrence of burrs in the peaks and valleys of the curve is due to the vibration generated by the braking at the extreme position.
Since the upper limb of the subject is offset from the standard position at the initial moment, the initial joint angle is not $0^{\circ}$.

B. Changes in joint angle and the hand coordinate system centre trajectory in the ADL experiment are shown in Figures 8, 9, 10and 11. 


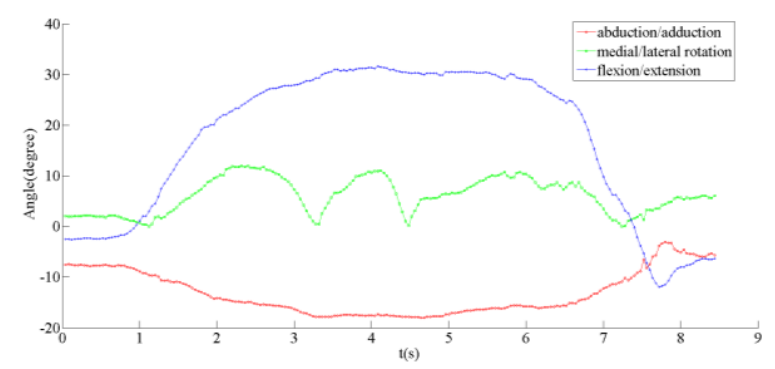

Figure 8: Shoulder joint angle of the ADL motion experiment: the shoulder flexes firstly forward about the z-axis of shoulder joint coordinate system, and it is accompanied by the tiny internal rotation of the shoulder joint when catching cap and pouring water.

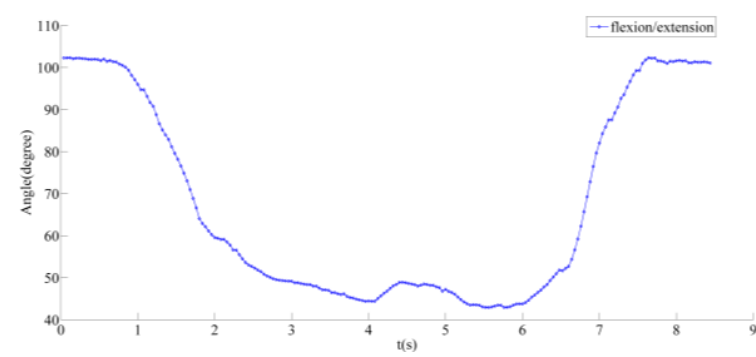

Figure 9: Elbow joint angle of the ADL motion experiment: it is accompanied by the flexion and extension of the elbow joint when the arm is extended forward to take the cup, and returns to the initial position when the pouring action is completed.

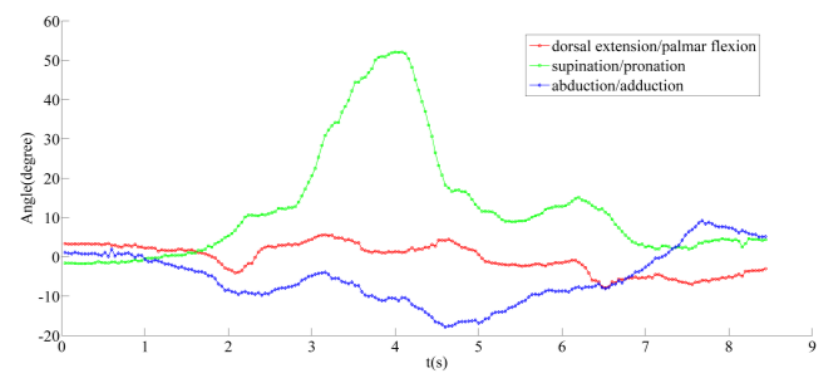

Figure 10: Wrist joint angle of the ADL motion experiment: the wrist joint rotates around the y-axis of wrist joint coordinate system at a certain angle to complete the pouring action.

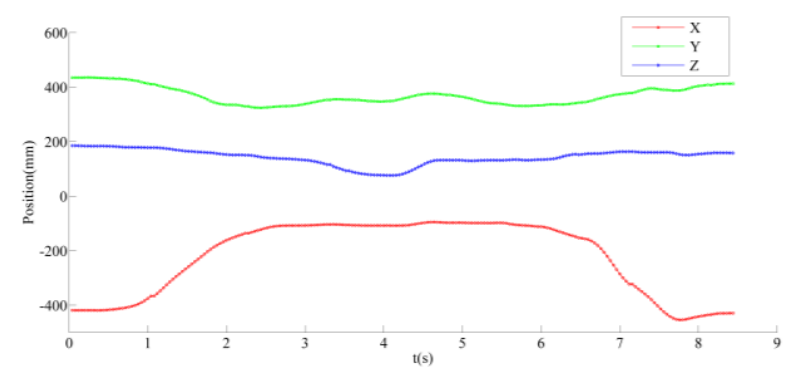

Figure 11: Position of the hand coordinate system centre of the ADL motion experiment: during the pouring process, the position of the hand coordinate system centre during the forward and backward movement of the arm leads to a significant change in the position in the $x$-axis direction and a small displacement in the $y$-axis and $z$-axis direction in the global coordinate system.

The main involved movements during the ADL experiment include flexion and extension of shoulder joint, flexion and extension of elbow joint, and pronation and supination of wrist joint. As can be seen from Figures 8, 9 and 10, the shoulder flexes forward around the z-axis of the shoulder coordinate system firstly, and the flexion and extension of elbow joint at the same time helps the upper limb to take a cup, and then the wrist joint pronates at a certain angle around the $y$-axis of the its joint coordinate system to complete the pouring action. Finally, the shoulder joint, the elbow joint, and the wrist joint return to the initial position sequentially. Figure 811 show that the result of the algorithm can accurately describe the kinematic parameters of the upper limb joints. 


\section{Conclusions}

The algorithm proposed by the combination of kinematic chain method and vector projection method to measure the kinematic parameters of upper limbs realizes the real-time measurement of the shoulder, elbow and wrist joint position and 7 DOF joint mobility, which is verified on the capture system and the results can meet the data requirements of quantitative evaluation of upper limbs. Compared with other upper limb kinematic parameters calculation methods, the measurement content is more complete. In the future, the method of this paper can be applied to the measurement of kinematics parameters in the lower limbs and other aspects, which will apply more quantitative description of the movement characteristics of the limb.

\section{Acknowledgements}

This research is supported by The National Key Research and Development Program of Chin (Grant No. 2018YFC2001601) and The Shanghai Municipal Science and Technology Commission (Grant No.18511108302).

\section{References}

[1] Cirstea, M. C. Compensatory strategies for reaching in stroke [J]. Brain, 2000, 123(5):940953.

[2] Wang Z, Chen L, Xie Q, et al. Upper Limb Hemiplegia Rehabilitation Robot Device With Adjustable Training Plan [J].

[3] Ashby P, Nailis A, Hunter J. The evaluation of spasticity [J]. Can J Neural Sci, 1987, 14(3):497500.

[4] Nguyen K D, Chen I M, Luo Z, et al. A Wearable Sensing System for Tracking and Monitoring of Functional Arm Movement [J]. IEEE/ASME Transactions on Mechatronics, 2011, 16(2):213220.

[5] Nurdiana Nordin, Sheng Quan Xie \& Burkhard Wünsche. Assessment of movement quality in robot- assisted upper limb rehabilitation after stroke: a review [J]. Journal of NeuroEngineering and Rehabilitation volume 11, Article number: 137 (2014).

[6] Zhou H, Hu H. Human motion tracking for rehabilitation-A survey [J]. Biomedical Signal Processing and Control, 2008, 3(1):1- 18.

[7] WANG M B. Rehabilitation Medicine [M]. Beijing: People's Medical Publishing House, 2009: 90.
[8] Chen J, Yang C J, Hofschulte J, et al. A robust optical/inertial data fusion system for motion tracking of the robot manipulator [J]. Frontiers of Information Technology \& Electronic Engineering, 2014, 15(7):574-583.

[9] AP Tsu, GM Abrams, NN Byl. Poststroke upper limb recovery [J]. Seminars in Neurology, 2014, 34(5): 485-495.

[10] Lu Zeng, Bingjin Ji, Xiao Li. A New AR-Based Framework for Wrist Assessment and Rehabilitation[C]. Proceeding of 2017 2nd Joint International Mechanical, Electronic and Information Technology Conference, 2017.

[11] Nguyen K D, Chen I M, Luo Z, et al. A Wearable Sensing System for Tracking and Monitoring of Functional Arm Movement [J]. IEEE/ASME Transactions on Mechatronics, 2011, 16(2):213220.

[12] Song H Y, Zhang J G, Wang F. Study on Motion Measurement of Human Upper Limb Based on Electromagnetic Tracking System during the Activities of Daily Living [J]. Applied Mechanics and Materials, 2013, 321-324:684-687.

[13] Han J J, Kurillo G, Abresch R T, et al. Upper extremity 3D reachable workspace analysis in dystrophinopathy using Kinect.[J]. Muscle \& Nerve, 2015, 52(3):344.

[14] Wei-Wei W, Yuan-Qi G, Hui G, et al. Measuring joint range of motion and evaluating measured results based on depth images [J]. Chinese Journal of Medical Physics, 2016.

[15] Kurillo G, Chen A, Bajcsy R, et al. Evaluation of upper extremity reachable workspace using Kinect camera [J]. Technology \& Health Care, 2013, 21(6):641-656.

[16] Chen W, Xiong C, Huang X, et al. Kinematic analysis and dexterity evaluation of upper extremity in activities of daily living [J]. Gait and Posture, 2010, 32(4):0-481.

[17] Pons J L. Wearable Robots: Biomechatronic Exoskeletons [M]. Hoboken, NJ, USA: Wiley, 2008.

[18] Mohammadzadeh F F, Liu S , Bond K A, et al. Feasibility of a Wearable, Sensor-based Motion Tracking System[J]. Procedia Manufacturing, 2015, 3:192-199.

[19] Elgohary M, Holmstrom L, Huisinga J, et al. Upper limb joint angle tracking with inertial sensors. [J]. Conf Proc IEEE Eng Med Biol Soc, 2011:5629-5632.

[20] Bai L , Pepper M G , Yan Y, et al. Quantitative Assessment of Upper Limb Motion in Neurorehabilitation Utilizing Inertial Sensors[J]. IEEE Transactions on Neural Systems and Rehabilitation Engineering, 2014, 23(2):232. 